Research Square

\title{
Hepatitis C Virus Viral Load And Mother-To-Child Transmission: a Systematic Review and Meta - Analysis
}

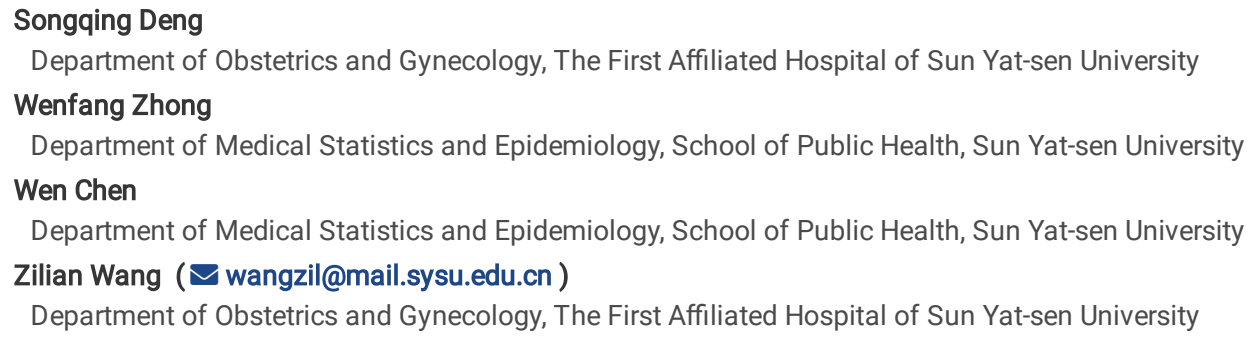

\section{Research Article}

Keywords: Hepatitis C virus, mother-to-child transmission, HCV viral load囚HIV

Posted Date: October 6th, 2021

DOI: https://doi.org/10.21203/rs.3.rs-929433/v1

License: () (1) This work is licensed under a Creative Commons Attribution 4.0 International License. Read Full License 


\section{Abstract}

Background: Hepatitis C virus (HCV) infection is increasing among pregnant women own to the opioid epidemic, and mother-to-child transmission (MTCT) was considered to be the leading cause of hepatitis $C$ in the future. Thus, our aim of this study was to assess the association of maternal HCV viral load and human immunodeficiency virus (HIV) coinfection with the risk of MTCT in pregnant women infected by HCV.

Methods: We performed a literature searching using MEDLINE, EMBASE, CENTRAL, SCIE, CPCIS, SCOPUS, LILACS and WHO Global Index Medicus from inception to 1 March 2021. Manuscripts were included if they reported the incidence of HCV MTCT. Pooled effect estimates were obtained by using randomeffects model and Holm-Bonferroni correction was performed for multiple pooled associations.

Results: a total of 26 studies involving 4491 newborns with maternal HCV infected were identified. Pregnant women with HCV viremia had increased risk of MTCT (odds ratio [OR]: 8.14, 95\% confidence interval [CI]: 4.62-14.31) compared with those with negative HCV-RNA. Multiple-subgroup analysis showed HCV viremia/HIV+ group experienced the highest risk for HCV MTCT and then HCV viremia mono-infected group; HCV-RNA negative women experienced the lowest risk for HCV MTCT. Among HCV viremia women, elevated risk of MTCT was found in subjects with viral load $\geq 6$ log copies/ml as compared with those with viral load $<6$ log copies/ml (OR=4.93, 95\% Cl: 2.38-10.21).

Conclusions: The HCV MTCT incidence is increased in pregnant women with detectable HCV viremia, and is even higher in those with viral load $\geq 6$ log copies/ml. HIV coinfection further increases the risk of HCV MTCT.

\section{Background}

Hepatitis $\mathrm{C}$ virus (HCV) infection is a major global health issue which affect $2-3 \%$ of population in the world ${ }^{1}$. HCV infection causes acute and chronic hepatitis, and increases the risk of liver cirrhosis, hepatocellular cancer, liver failure ${ }^{2,3}$. In the last decade, the prevalence of HCV in reproductive aged women was increasing own to opioid use ${ }^{4}$, resulting in a dramatic rise of $\mathrm{HCV}$ infection among pregnant women ${ }^{5}$. HCV viremia were detected in $30-40 \%$ infected neonates within the first three days of life, indicated that intrauterine transmission may occur ${ }^{6}$. Therefore, mother-to-child transmission (MTCT) was considered to be the leading cause of hepatitis $\mathrm{C}$ in the future ${ }^{7}$, and current guidelines recommend universal HCV screening during pregnancy 8,9 .

Antiviral treatment is considered as proven interventions to decrease the risk of perinatal transmission and neonatal infection, such as human immunodeficiency virus (HIV) and hepatitis B virus infection during pregnancy ${ }^{10,11}$. For HCV antiviral treatment, direct-acting antiviral (DAA) regimens are able to reduce $\mathrm{HCV}$ viremia to undetectable level within two weeks ${ }^{12,13}$. Although it was recently reported effective to reduce HCV-RNA titer in pregnant women with DAA regime ${ }^{14}$, there are no epidemiologic studies or clinical trials have confirmed the reduction of HCV viral load in pregnancy contributes to the prevention of MTCT.

Although with some debates, HCV viremia has been considered as a main risk factor of MTCT during pregnancy ${ }^{15}$. Existing studies show when the viral load greater than or equal to $6 \log 10$ copies/ml, the risk of MTCT is higher than women with a lower viral load ${ }^{16-18}$. Placenta express multiple HCV-receptors and/or entry cofactors (e.g., claudin-1, occludin, SR-B1, LDLr or DC-SIGN), resulting in HCV transmission of placenta barrier and fetal infection ${ }^{19,20}$. In addition, exposure to maternal infected blood during birth is another important pathway of HCV vertical transmission ${ }^{21}$, potentially because of peripheral mononuclear cells infection of $\mathrm{HCV}^{20}$. However, some clinical studies did not support this hypothesis ${ }^{22,23}$. For example, Jhaveri et al. found HCV MTCT even when maternal HCV RNA load below $200001 \mathrm{U} / \mathrm{ml}^{22}$.

Injection drug use and the opioid epidemic is the leading cause of both HCV and HIV infection in the last decades ${ }^{24}$, and $\mathrm{HIV} / \mathrm{HCV}$ co-infection is frequently observed in HCV pregnant women ${ }^{25}$. The most recent systematic review and meta-analysis showed maternal HIV coinfection is a main risk for MTCT of HCV. HIV down regulates the immune responses, in particular of the T cell mediated reactivity with expansion of Treg cells ${ }^{19}$. In immunodeficiency status, HCV replication may be increased and therefore facilitate transmission ${ }^{7,26}$, and it is suggested that HIV antiviral treatment may prevent HCV MTCT. However, whether HCV-viremia mono-infection or HCV-viremia coinfection of HIV increases MTCT of HCV has not been fully clarified.

In this study, therefore, we aim to compare the risk of MTCT of HCV between pregnant women with and without HCV viremia detected in circulation, and to study how HCV viral load associated with MTCT did. In addition, the relationship of HIV coinfection with MTCT was investigated in the current meta-analysis.

\section{Methods}

\section{Search strategy and selection criteria}

We conducted this systematic review according to a protocol registered in PROSPERO (CRD42021239499), available at: https://www.crd.york.ac.uk/PROSPERO/. A comprehensive search of MEDLINE, EMBASE, the Cochrane Central Register of Controlled Trials (CENTRAL), Science Citation Index Expanded (SCIE), Conference Proceedings Citation Index-Science (CPCIS), SCOPUS, Literature Latino-Americana e do Caribe em Ciências da Saúde (LILACS) and WHO Global Index Medicus was conducted from database inception to January 7, 2021. Detailed search strategy and search terms are available in supplementary Table 1.

To minimize differences between studies, we imposed the following methodological restrictions for the inclusion criteria: (i) study designs: cohort studies or case-control studies, (ii) including data on both anti-HCV positive mothers and their children with documented HCV serology, (iii) comparing MTCT incidence between HCV viremia women and those with negative HCV-RNA. MTCT was confirmed when HCV-RNA was detected in newborns. In instances of multiple 
publications, the most up-to-date or comprehensive information was used. Citations initially selected by systematic search were first retrieved as a title or abstract and preliminarily screened. Potentially suitable citations were then retrieved as complete manuscripts and assessed for compliance to inclusion criteria.

\section{Data extraction}

Articles were reviewed and crosschecked independently by two authors. Data on the following characteristics were independently extracted: first author, publication year, county, study type, year of enrollment, number of mother-child pairs, maternal age of delivery, HCV-RNA detection method, number of live births, duration and frequency of newborns follow-up, HCV MTCT incidence, maternal HCV-RNA titer, and crude numbers of newborn with HCV infected and not. HCV MTCT incidence was defined as the ratio of the number of infected newborns to the total number of newborns. High level of viremia was defined as the HCV-RNA titers greater than or equal to $6 \log 10 \mathrm{copies} / \mathrm{ml}^{16}$. The most comprehensive data were included if multiple articles were reported on the overlapping study population. Any disagreements were resolved by consensus.

\section{Quality assessments}

We used the Newcastle-Ottawa Scale (NOS) to evaluate the methodological quality of the included observational studies. NOS includes three dimensions to assess the overall quality and a maximum of 4,2 , and 3 stars can be rated for selection, comparability, and outcome assessment respectively. Two authors independently assessed the risk of bias of included studies. Study scored $\leq 5$ on the NOS was regarded as being of low quality and was excluded 27 .

\section{Statistical analyses}

We assessed the risk of the HCV MTCT by conducting two meta-analysis to calculate the odds ratios (ORs) and 95\% confidence intervals (Cls) for individual studies and then pool the estimates using the DerSimonian and Laird random-effects models. First, we compared the HCV MTCT risk between HCV viremia women and those with negative HCV-RNA. Then, we performed a meta-analysis for relationship between HCV-RNA titers and HCV MTCT incidence.

Multiple-subgroup analysis among maternal HCV viremia/HIV+ group, HCV viremia mono-infected group and HCV-RNA negative group was further conducted after the comparison between HCV viremia and negative HCV-RNA women. In order to control the increased type I errors triggered by multiple comparison ${ }^{28}$, Holm-Bonferroni method ${ }^{29}$ was used to figure out adjusted $P$-value.

We used the $\chi^{2}$-based $Q$ test to assess heterogeneity across studies, expressed as Cochran's $Q$ and $P$ statistics, together with $95 \%$ confidence intervals. We also determine the possibility of publication bias by using the funnel plot, Begg's test, and Egger's test. Sensitivity analyses were further conducted by only included prospective studies with a sample size larger than 100. Subgroup analysis was conducted by country income according to the World Bank's list of economies ${ }^{30}$, and region of different incidence of $\mathrm{HCV}$ infection according to the WHO Global Hepatitis Report ${ }^{23}$. Lower middle income countries and upper middle income countries were further aggregated into middle income countries. We also undertook meta-regression by publication year and MTCT incidence.

All statistical analyses were performed using the software $\mathrm{R}$ version 4.0.2.

\section{Results}

\section{Characteristics of included studies}

We identified 717 papers and 202 of which were removed due to duplications. Finally, 26 publications, published between 1993 and 2015 , were eligible for inclusion (Figure 1). The studies examined 4491 newborns of HCV infected mother, with a follow up of at least 6 months. There were 3 retrospective cohorts and 23 prospective cohorts. Of the primary studies, 100\% had described independent, consecutive sampling of their cohort. All studies detected HCV-RNA by polymerase chain reaction, and the MTCT incidence ranged from $1.3-38.5 \%$.

Of the twenty-six studies, twenty-three independent eligible studies evaluated the risk of HCV MTCT between maternal HCV viremia group and negative HCVRNA group (Table 1). Thirteen independent eligible studies evaluated the risk of HCV MTCT between HCV viremia/ HIV+ co-infected mothers and HCV viremia mono-infected mothers (Table 1). Nine independent eligible studies estimated the risk of HCV MTCT based on different HCV-RNA titers (Table 2). 
Table 1

Characteristics of studies assessing maternal HCV-RNA and MTCT risk.

\begin{tabular}{|c|c|c|c|c|c|c|c|c|c|c|c|c|}
\hline \multirow[t]{2}{*}{ ID } & \multirow[t]{2}{*}{$\begin{array}{l}\text { First } \\
\text { author }\end{array}$} & \multirow[t]{2}{*}{ Year } & \multirow[t]{2}{*}{ Country } & \multirow[t]{2}{*}{$\begin{array}{l}\text { Study } \\
\text { design }\end{array}$} & \multirow[t]{2}{*}{$\begin{array}{l}\text { Year of } \\
\text { enrollment }\end{array}$} & \multirow{2}{*}{$\begin{array}{l}\text { Mother- } \\
\text { child } \\
\text { pairs, n }\end{array}$} & \multirow{2}{*}{$\begin{array}{l}\text { Mother } \\
\text { age, } \\
\text { years }\end{array}$} & \multirow{2}{*}{$\begin{array}{l}\text { HCV- } \\
\text { RNA } \\
\text { detection } \\
\text { method }\end{array}$} & \multirow{2}{*}{$\begin{array}{l}\text { Total no. } \\
\text { of } \\
\text { newborns }\end{array}$} & \multirow[t]{2}{*}{ Follow-up } & \multirow{2}{*}{$\begin{array}{l}\text { HCV } \\
\text { MTCT } \\
\text { incidence, } \\
\%\end{array}$} & \multirow{2}{*}{$\begin{array}{l}\begin{array}{l}\text { Materne } \\
\text { RNA (+) }\end{array} \\
\begin{array}{l}\text { MTCT } \\
(+)\end{array}\end{array}$} \\
\hline & & & & & & & & & & & & \\
\hline 2 & $\begin{array}{l}\text { Saez A et } \\
\text { al. }\end{array}$ & 2004 & Italy & prospective & $\begin{array}{l}1992.1- \\
2000.12\end{array}$ & 119 & $\begin{array}{l}32(16 \\
-48)\end{array}$ & PCR & 122 & $\begin{array}{l}0,6,12,24, \\
36 \text { months } \\
\text { from } \\
\text { delivery }\end{array}$ & $1.6 \%$ & 2 \\
\hline 3 & $\begin{array}{l}\text { Elmagd } \\
\text { EKA et al. }\end{array}$ & 2011 & Egypt & retrospective & NR & 8 & $20-40$ & PCR & 8 & $\begin{array}{l}\text { up to } 12 \\
\text { months } \\
\text { after } \\
\text { delivery }\end{array}$ & $25.0 \%$ & 2 \\
\hline 5 & $\begin{array}{l}\text { Mariné- } \\
\text { Barjoan E } \\
\text { et al. }\end{array}$ & 2007 & France & prospective & $\begin{array}{l}1998.10- \\
2002.9\end{array}$ & 214 & $\begin{array}{l}34(30 \\
-37)\end{array}$ & PCR & 214 & $\begin{array}{l}\text { at birth and } \\
\text { at } 3,6 \text { and } \\
12\end{array}$ & $6.1 \%$ & 12 \\
\hline 9 & $\begin{array}{l}\text { Granovsky } \\
\text { MO et al. }\end{array}$ & 1998 & America & prospective & $\begin{array}{l}\text { 1986.1- } \\
1991.1\end{array}$ & NR & NR & PCR & 122 & $\begin{array}{l}\text { up to } 4 \\
\text { years after } \\
\text { delivery }\end{array}$ & $6.7 \%$ & 6 \\
\hline 11 & $\begin{array}{l}\text { Ngo-Giang- } \\
\text { Huong N et } \\
\text { al. }\end{array}$ & 2010 & Thailand & prospective & $\begin{array}{l}1997.6- \\
1999.12\end{array}$ & 41 & NR & PCR & 41 & $\begin{array}{l}\text { at between } \\
6 \text { weeks } \\
\text { and } 6 \\
\text { months }\end{array}$ & $9.8 \%$ & 4 \\
\hline 12 & $\begin{array}{l}\text { Steininger } \\
\text { C et al. }\end{array}$ & 2003 & Australia & retrospective & $\begin{array}{l}1994- \\
1999\end{array}$ & 73 & NR & PCR & 75 & NR & $12.0 \%$ & 9 \\
\hline 18 & $\begin{array}{l}\text { Dal Molin } \\
\text { G et al. }\end{array}$ & 2002 & Italy & prospective & $\begin{array}{l}1994.1- \\
2000.12\end{array}$ & 105 & $\begin{array}{l}30.25 \\
\pm 4.95\end{array}$ & PCR & 105 & $\begin{array}{l}\text { at } 1 \text { month, } \\
3-4 \\
\text { months, } \\
\text { and } 8-13 \\
\text { months of } \\
\text { age }\end{array}$ & $4.8 \%$ & 5 \\
\hline 20 & $\begin{array}{l}\text { Money Det } \\
\text { al. }\end{array}$ & 2014 & Columbia & prospective & $\begin{array}{l}2000- \\
2003\end{array}$ & 117 & $\begin{array}{l}30(26 \\
-34)\end{array}$ & PCR & 117 & $\begin{array}{l}\text { at three, } \\
\text { six, } 12 \text {, and } \\
18 \text { months } \\
\text { after birth }\end{array}$ & $2.8 \%$ & 4 \\
\hline 26 & $\begin{array}{l}\text { Tajiri H et } \\
\text { al. }\end{array}$ & 2001 & Japan & prospective & $\begin{array}{l}1993 \text { to } \\
1998\end{array}$ & 114 & NR & PCR & 114 & $\begin{array}{l}\text { at } 0,3,6,9 \text {, } \\
12 \text { months } \\
\text { and every } \\
\text { year } \\
\text { thereafter }\end{array}$ & $7.9 \%$ & 9 \\
\hline 27 & $\begin{array}{l}\text { Mazza C et } \\
\text { al. }\end{array}$ & 1998 & Italy & prospective & $\begin{array}{l}1994.1- \\
1996.1\end{array}$ & 70 & $\begin{array}{l}29.8 \pm \\
5.3\end{array}$ & PCR & 70 & $\begin{array}{l}\text { was first } \\
\text { detected } \\
\text { between } 2 \\
\text { and } 6 \\
\text { months }\end{array}$ & $8.0 \%$ & 6 \\
\hline 28 & $\begin{array}{l}\text { Ferrero S } \\
\text { et al. }\end{array}$ & 2003 & Italy & prospective & $\begin{array}{l}1990.3- \\
2000.3\end{array}$ & 170 & $\begin{array}{l}30.8 \pm \\
5.3\end{array}$ & PCR & 188 & $\begin{array}{l}\text { at birth and } \\
\text { at } 3,6,9 \\
12 \text { and } 18 \\
\text { months of } \\
\text { age }\end{array}$ & $2.7 \%$ & 5 \\
\hline 31 & $\begin{array}{l}\text { Murakami } \\
\text { J et al. }\end{array}$ & 2012 & Japan & prospective & $\begin{array}{l}\text { "1992.6- } \\
1998.12\end{array}$ & NR & $\begin{array}{l}31.2 \pm \\
4.0\end{array}$ & PCR & 125 & $\begin{array}{l}\text { at least } 6 \\
\text { months } \\
\text { after } \\
\text { delivery }\end{array}$ & $8.8 \%$ & 11 \\
\hline 32 & $\begin{array}{l}\text { Mast EE et } \\
\text { al. }\end{array}$ & 2005 & America & prospective & $\begin{array}{l}\text { 1993.11- } \\
1996.7\end{array}$ & 242 & NR & PCR & 244 & $\begin{array}{l}\text { at } 1,2,4,6 \text {, } \\
9,12,15, \\
18, \text { and } 24 \\
\text { months of } \\
\text { age }\end{array}$ & $3.7 \%$ & 9 \\
\hline 33 & $\begin{array}{l}\text { Garcia- } \\
\text { Tejedor A } \\
\text { et al. }\end{array}$ & 2015 & Spain & retrospective & $\begin{array}{l}1986- \\
2011\end{array}$ & 710 & NR & PCR & 711 & $\begin{array}{l}\text { at } 1,3,6,9 \text {, } \\
12,15,18 \\
\text { and } 24 \\
\text { months of } \\
\text { age }\end{array}$ & $2.7 \%$ & 12 \\
\hline
\end{tabular}




\begin{tabular}{|c|c|c|c|c|c|c|c|c|c|c|c|c|}
\hline \multirow[t]{2}{*}{ ID } & \multirow[t]{2}{*}{$\begin{array}{l}\text { First } \\
\text { author }\end{array}$} & \multirow[t]{2}{*}{ Year } & \multirow[t]{2}{*}{ Country } & \multirow[t]{2}{*}{$\begin{array}{l}\text { Study } \\
\text { design }\end{array}$} & \multirow[t]{2}{*}{$\begin{array}{l}\text { Year of } \\
\text { enrollment }\end{array}$} & \multirow{2}{*}{$\begin{array}{l}\text { Mother- } \\
\text { child } \\
\text { pairs, } n\end{array}$} & \multirow{2}{*}{$\begin{array}{l}\text { Mother } \\
\text { age, } \\
\text { years }\end{array}$} & \multirow{2}{*}{$\begin{array}{l}\text { HCV- } \\
\text { RNA } \\
\text { detection } \\
\text { method }\end{array}$} & \multirow{2}{*}{$\begin{array}{l}\text { Total no. } \\
\text { of } \\
\text { newborns }\end{array}$} & \multirow[t]{2}{*}{ Follow-up } & \multirow{2}{*}{$\begin{array}{l}\text { HCV } \\
\text { MTCT } \\
\text { incidence, } \\
\%\end{array}$} & \multirow{2}{*}{$\begin{array}{l}\text { Materne } \\
\text { RNA (+) } \\
\underset{\text { MTCT }}{(+)}\end{array}$} \\
\hline & & & & & & & & & & & & \\
\hline 43 & $\begin{array}{l}\text { Ruiz- } \\
\text { Exxtremera } \\
\text { Á et al. }\end{array}$ & 2013 & Spain & prospective & $\begin{array}{l}1991- \\
2009\end{array}$ & 122 & NR & PCR & 122 & $\begin{array}{l}\text { at birth and } \\
\text { at } 2,4,6,8, \\
10,12,18 \\
\text { and } 24 \\
\text { months, } \\
\text { and } \\
\text { thereafter } \\
\text { at } 3,4,5 \\
\text { and } 6 \\
\text { years. }\end{array}$ & $5.6 \%$ & 15 \\
\hline 40 & $\begin{array}{l}\text { OHTO H et } \\
\text { al. }\end{array}$ & 1994 & Japan & prospective & $\begin{array}{l}1990.5- \\
1992.12\end{array}$ & 54 & NR & PCR & 54 & $\begin{array}{l}\text { at birth and } \\
\text { were } \\
\text { followed } \\
\text { for at least } \\
6 \text { months } \\
\text { after birth }\end{array}$ & $6.7 \%$ & 3 \\
\hline 42 & $\begin{array}{l}\text { Spence JD } \\
\text { et al. }\end{array}$ & 1997 & Australia & prospective & $\begin{array}{l}1995- \\
1997\end{array}$ & 125 & NR & PCR & 125 & $\begin{array}{l}\text { at birth and } \\
\text { at } 6,12,18 \\
\text { and }>18 \\
\text { months of } \\
\text { age }\end{array}$ & $12.3 \%$ & 6 \\
\hline 45 & $\begin{array}{l}\text { Ceci } O \text { et } \\
\text { al. }\end{array}$ & 2001 & Italy & prospective & $\begin{array}{l}1995.1- \\
1997.6\end{array}$ & 78 & $\begin{array}{l}30(21 \\
-42)\end{array}$ & PCR & 78 & $\begin{array}{l}\text { at birth, } 4 \text {, } \\
8,12,18 \\
\text { and } 24 \\
\text { months of } \\
\text { age }\end{array}$ & $38.5 \%$ & 30 \\
\hline 49 & $\begin{array}{l}\text { Resti M et } \\
\text { al. }\end{array}$ & 1998 & Italy & prospective & NR & 403 & NR & PCR & 403 & $\begin{array}{l}\text { at birth and } \\
\text { then at } \\
\text { least three } \\
\text { times } \\
\text { during the } \\
\text { follow up }\end{array}$ & $3.2 \%$ & 13 \\
\hline 50 & $\begin{array}{l}\text { Zuccotti } \\
\text { GV et al. }\end{array}$ & 1995 & Italy & prospective & $\begin{array}{l}\text { 1991.1- } \\
1993.1\end{array}$ & 37 & $\begin{array}{l}26(19 \\
-33)\end{array}$ & PCR & 37 & $\begin{array}{l}\text { at birth and } \\
\text { at } 1 \text { month } \\
\text { of age, and } \\
\text { then every } \\
3 \text { months } \\
\text { until } 18 \\
\text { months of } \\
\text { age }\end{array}$ & $16.2 \%$ & 6 \\
\hline 53 & $\begin{array}{l}\text { Zanetti AR } \\
\text { et al. }\end{array}$ & 1998 & Italy & prospective & 1990 & 291 & NR & PCR & 291 & $\begin{array}{l}\text { at birth and } \\
\text { at } 3,6,9,12 \\
\text { months of } \\
\text { age and } \\
\text { then every } \\
6 \text { months. }\end{array}$ & $27.0 \%$ & 17 \\
\hline 52 & $\begin{array}{l}\text { Paccagnini } \\
\text { S. }\end{array}$ & 1995 & Italy & prospective & 1990 & 70 & NR & NR & 70 & $\begin{array}{l}\text { at } 0,3,6,9, \\
12,15 \text { and } \\
18 \text { months }\end{array}$ & $8.2 \%$ & 9 \\
\hline 54 & $\begin{array}{l}\text { Resti M et } \\
\text { al. }\end{array}$ & 2002 & Italy & prospective & $\begin{array}{l}1993.4- \\
1996.12\end{array}$ & 1372 & NR & PCR & 1372 & $\begin{array}{l}\text { at birth or } \\
\text { within } 3 \\
\text { months } \\
\text { and then } \\
>3 \text { times } \\
\text { during the } \\
\text { subsequent } \\
2 \text {-year } \\
\text { follow-up } \\
\text { period. }\end{array}$ & $7.6 \%$ & 97 \\
\hline
\end{tabular}


Table 1

(Continued)

\begin{tabular}{|c|c|c|c|c|c|}
\hline \multirow[t]{2}{*}{ ID } & \multirow[t]{2}{*}{ First author } & \multicolumn{2}{|c|}{ Maternal HCV viraemic/HIV+ co-infection } & \multicolumn{2}{|c|}{ Maternal HCV viraemic/HIV- } \\
\hline & & MTCT (+) & МТст (-) & MTCT (+) & МтСТ (-) \\
\hline 2 & Saez A et al. & NR & NR & NR & NR \\
\hline 3 & Elmagd EKA et al. & NR & NR & NR & NR \\
\hline 5 & Mariné-Barjoan E et al. & NR & NR & NR & NR \\
\hline 9 & Granovsky $\mathrm{MO}$ et al. & 4 & 43 & 2 & 23 \\
\hline 11 & Ngo-Giang-Huong $\mathrm{N}$ et al. & NR & NR & NR & NR \\
\hline 12 & Steininger $\mathrm{C}$ et al. & NR & NR & NR & NR \\
\hline 18 & Dal Molin G et al. & NR & NR & NR & NR \\
\hline 20 & Money Det al. & 0 & 15 & 4 & 89 \\
\hline 26 & Tajiri H et al. & NR & NR & NR & NR \\
\hline 27 & Mazza C et al. & NR & NR & NR & NR \\
\hline 28 & Ferrero $\mathrm{S}$ et al. & 2 & 28 & 3 & 102 \\
\hline 31 & Murakami J et al. & NR & NR & NR & NR \\
\hline 32 & Mast EE et al. & 2 & 6 & 7 & 175 \\
\hline 33 & Garcia-Tejedor A et al. & NR & NR & NR & NR \\
\hline 43 & Ruiz-Extremera Á et al. & NR & NR & NR & NR \\
\hline 40 & OHTO H et al. & NR & NR & NR & NR \\
\hline 42 & Spence JD et al. & NR & NR & NR & NR \\
\hline 45 & Ceci $O$ et al. & NR & NR & NR & NR \\
\hline 49 & Resti M et al. & NR & NR & NR & NR \\
\hline 50 & Zuccotti GV et al. & 4 & 9 & 2 & 6 \\
\hline 53 & Zanetti AR et al. & 9 & 23 & 8 & 167 \\
\hline 52 & Paccagnini S. & 7 & 10 & 2 & 4 \\
\hline 54 & Resti M et al. & 22 & 136 & 75 & 664 \\
\hline
\end{tabular}


Table 2

Characteristics of the included studies assessing HCV viral load and MTCT risk

\begin{tabular}{|c|c|c|c|c|c|c|c|c|c|c|c|c|}
\hline ID & $\begin{array}{l}\text { First } \\
\text { author }\end{array}$ & Year & Country & $\begin{array}{l}\text { Study } \\
\text { design }\end{array}$ & $\begin{array}{l}\text { Year of } \\
\text { enrollment }\end{array}$ & $\begin{array}{l}\text { Mother- } \\
\text { child } \\
\text { pairs, n }\end{array}$ & $\begin{array}{l}\text { Mother } \\
\text { age, } \\
\text { years }\end{array}$ & $\begin{array}{l}\text { HCV- } \\
\text { RNA } \\
\text { detection } \\
\text { method }\end{array}$ & $\begin{array}{l}\text { Total no. } \\
\text { of } \\
\text { newborns }\end{array}$ & $\begin{array}{l}\text { Follow- } \\
\text { up }\end{array}$ & $\begin{array}{l}\text { HCV } \\
\text { MTCT } \\
\text { incidence, } \\
\%\end{array}$ & $\begin{array}{l}\text { HCV-RNA } \\
\text { category, } \\
\log _{10} \\
\text { copies/mL }\end{array}$ \\
\hline \multirow[t]{2}{*}{2} & $\begin{array}{l}\text { Saez A } \\
\text { et al. }\end{array}$ & 2004 & Italy & prospective & $\begin{array}{l}1992.1- \\
2000.12\end{array}$ & 80 & $\begin{array}{l}32(16- \\
48)\end{array}$ & PCR & 80 & $\begin{array}{l}\text { at birth } \\
\text { and at } 6, \\
12,24 \\
\text { and } 36 \\
\text { months } \\
\text { of age }\end{array}$ & $2.5 \%$ & $\begin{array}{l}<10^{6} \\
\text { copies/mL }\end{array}$ \\
\hline & & & & & & & & & & & & $\begin{array}{l}\geq 10^{6} \\
\text { copies } / \mathrm{mL}\end{array}$ \\
\hline \multirow[t]{2}{*}{5} & $\begin{array}{l}\text { Mariné- } \\
\text { Barjoan } \\
\text { E et al. }\end{array}$ & 2007 & France & prospective & $\begin{array}{l}1998.10- \\
2002.9\end{array}$ & 194 & $\begin{array}{l}34(30- \\
37)\end{array}$ & PCR & 194 & $\begin{array}{l}\text { at birth } \\
\text { and at } 3, \\
6 \text { and } \\
12 \\
\text { months } \\
\text { of age }\end{array}$ & $6.2 \%$ & $\begin{array}{l}<10^{6} \\
\text { copies/mL }\end{array}$ \\
\hline & & & & & & & & & & & & $\begin{array}{l}\geq 10^{6} \\
\text { copies/mL }\end{array}$ \\
\hline \multirow[t]{2}{*}{22} & $\begin{array}{l}\text { Lin } \mathrm{HH} \text { et } \\
\text { al. }\end{array}$ & 1994 & China & prospective & $\begin{array}{l}1989.3- \\
1992.4\end{array}$ & 8 & NR & PCR & 8 & $\begin{array}{l}\text { at I } \\
\text { week } \\
\text { and } 1,3 \text {, } \\
6,9 \text {, and } \\
12 \\
\text { months } \\
\text { of age }\end{array}$ & $12.5 \%$ & $\begin{array}{l}<10^{6} \\
\text { copies/mL }\end{array}$ \\
\hline & & & & & & & & & & & & $\begin{array}{l}\geq 10^{6} \\
\text { copies/mL }\end{array}$ \\
\hline \multirow[t]{2}{*}{25} & $\begin{array}{l}\text { Okamoto } \\
\text { M et al. }\end{array}$ & 2000 & Japan & prospective & $\begin{array}{l}\text { 1992.6- } \\
1998.12\end{array}$ & 84 & NR & PCR & 84 & NR & $8.3 \%$ & $\begin{array}{l}<10^{6} \\
\text { copies } / \mathrm{mL}\end{array}$ \\
\hline & & & & & & & & & & & & $\begin{array}{l}\geq 10^{6} \\
\text { copies/mL }\end{array}$ \\
\hline \multirow[t]{2}{*}{32} & $\begin{array}{l}\text { Mast EE } \\
\text { et al. }\end{array}$ & 2005 & America & prospective & $\begin{array}{l}\text { 1993.11- } \\
1996.7\end{array}$ & 182 & NR & PCR & 182 & $\begin{array}{l}\text { at } 1,2 \text {, } \\
4,6,9, \\
12,15, \\
18, \text { and } \\
24 \\
\text { months } \\
\text { of age }\end{array}$ & $3.8 \%$ & $\begin{array}{l}\leq 10^{6} \\
\text { copies/ml }\end{array}$ \\
\hline & & & & & & & & & & & & $\begin{array}{l}>10^{6} \\
\text { copies } / \mathrm{ml}\end{array}$ \\
\hline \multirow[t]{2}{*}{33} & $\begin{array}{l}\text { Garcia- } \\
\text { Tejedor } \\
\text { A et al. }\end{array}$ & 2015 & Spain & retrospective & $\begin{array}{l}1986- \\
2011\end{array}$ & 227 & NR & NR & 227 & $\begin{array}{l}\text { at } 1,3, \\
6,9,12, \\
15,18 \\
\text { and } 24 \\
\text { months } \\
\text { of age }\end{array}$ & $1.3 \%$ & $\begin{array}{l}<10^{6} \\
\text { copies/ml }\end{array}$ \\
\hline & & & & & & & & & & & & $\begin{array}{l}\geq 10^{6} \\
\text { copies/ml }\end{array}$ \\
\hline \multirow[t]{2}{*}{39} & $\begin{array}{l}\text { Ketzinel- } \\
\text { Gilad M } \\
\text { et al. }\end{array}$ & 2000 & Israel & prospective & $\begin{array}{l}1992- \\
1997\end{array}$ & 17 & $23-41$ & PCR & 17 & $\begin{array}{l}\text { at } 2 \\
\text { days } \\
\text { after } \\
\text { birth } \\
\text { and up } \\
\text { to } 4 \\
\text { years }\end{array}$ & $11.8 \%$ & $\begin{array}{l}<10^{6} \\
\text { copies/mL }\end{array}$ \\
\hline & & & & & & & & & & & & $\begin{array}{l}\geq 10^{6} \\
\text { copies/mL }\end{array}$ \\
\hline
\end{tabular}




\begin{tabular}{|c|c|c|c|c|c|c|c|c|c|c|c|c|}
\hline ID & $\begin{array}{l}\text { First } \\
\text { author }\end{array}$ & Year & Country & $\begin{array}{l}\text { Study } \\
\text { design }\end{array}$ & $\begin{array}{l}\text { Year of } \\
\text { enrollment }\end{array}$ & $\begin{array}{l}\text { Mother- } \\
\text { child } \\
\text { pairs, } n\end{array}$ & $\begin{array}{l}\text { Mother } \\
\text { age, } \\
\text { years }\end{array}$ & $\begin{array}{l}\text { HCV- } \\
\text { RNA } \\
\text { detection } \\
\text { method }\end{array}$ & $\begin{array}{l}\text { Total no. } \\
\text { of } \\
\text { newborns }\end{array}$ & $\begin{array}{l}\text { Follow- } \\
\text { up }\end{array}$ & $\begin{array}{l}\text { HCV } \\
\text { MTCT } \\
\text { incidence, } \\
\%\end{array}$ & $\begin{array}{l}\text { HCV-RNA } \\
\text { category, } \\
\log _{10} \\
\text { copies/mL }\end{array}$ \\
\hline \multirow[t]{2}{*}{40} & $\begin{array}{l}\text { OHTO H } \\
\text { et al. }\end{array}$ & 1994 & Japan & prospective & $\begin{array}{l}1990.5- \\
1992.12\end{array}$ & 37 & NR & PCR & 37 & $\begin{array}{l}\text { at birth } \\
\text { and } \\
\text { were } \\
\text { followed } \\
\text { for at } \\
\text { least } 6 \\
\text { months } \\
\text { after } \\
\text { birth }\end{array}$ & $18.9 \%$ & $\begin{array}{l}<10^{6} \\
\text { copies/mL }\end{array}$ \\
\hline & & & & & & & & & & & & $\begin{array}{l}\geq 10^{6} \\
\text { copies } / \mathrm{mL}\end{array}$ \\
\hline \multirow[t]{2}{*}{42} & $\begin{array}{l}\text { Spence } \\
\text { JD et al. }\end{array}$ & 1997 & Australia & prospective & $\begin{array}{l}1995- \\
1997\end{array}$ & 52 & NR & PCR & 52 & $\begin{array}{l}\text { at birth } \\
\text { and at } 6 \text {, } \\
12,18 \\
\text { and }>18 \\
\text { months } \\
\text { of age }\end{array}$ & $11.5 \%$ & $\begin{array}{l}<10^{6} \\
\text { copies/mL }\end{array}$ \\
\hline & & & & & & & & & & & & $\begin{array}{l}\geq 10^{6} \\
\text { copies } / \mathrm{mL}\end{array}$ \\
\hline
\end{tabular}

Notes: NR: not reported.

All of the 26 studies included in the present meta-analysis were regarded as acceptable quality, with NOS scores between 6 and 9. (supplementary Table 2, Table 3.)

\section{Comparison of HCV MTCT risk between maternal HCV viremia and negative HCV-RNA group}

Twenty-three publications ${ }^{17,18,31-51}$ with 4382 newborns of HCV infected mother and 296 MTCT children were identified. The pooled MTCT incidence in the maternal HCV viremia group was 9.55\% (95\% Cl: 8.55\%-10.64\%), compared with $0.30 \%$ (95\% Cl: 0.11\%-0.80\%) in negative HCV-RNA group (Table 1).

Heterogeneity was not presented in the included studies $(\mathrm{Q}=8.21, P=1.000 ; P=0.00 \%)$. The pooled OR of MTCT in maternal HCV viremia compared to negative HCV-RNA group was $8.14(95 \% \mathrm{Cl}, 4.62-14.31 ; P<0.0001)$ by random-effects model (Fig. $2 \mathrm{~A})$. A sensitivity analysis including only prospective studies with a sample size larger than 100 showed the pooled OR of MTCT was $8.83(95 \% \mathrm{Cl}: 4.18-18.65)$, indicating good robustness of the results (supplementary appendix Fig. 1A)

The funnel plot showed symmetric distribution which indicated the absence of publication bias (supplementary appendix Fig. 2A). However, Begg's test $(P=$ $0.010)$ and Egger's test $(P=0.357)$ suggested the existence of publication bias.

Multiple-subgroup analysis was conducted among maternal HCV viremia/HIV+ group, HCV viremia mono-infected group and HCV-RNA negative group. The pooled OR was 2.33 (95\% Cl: 1.16-4.68) for MTCT in HCV viremia/ HIV+ versus HCV viremia mono-infected group, 8.16 (95\% Cl: 3.19-20.88) for HCV viremia mono-infected versus HCV-RNA negative group, 15.41(95\% Cl: 5.69-41.74) for HCV viremia/ HIV+ versus HCV-RNA negative group, respectively. All the three significant pooled ORs survived Holm-Bonferroni correction (adjusted $P$ values are $0.0174,0.0003$ and 0.0003 respectively) (Fig. 2A).

Subgroup analysis by country income showed the pooled OR of MTCT was 8.79 (95\% Cl: $4.85-15.95)$ in the high-income country subgroup, and 4.06 ( $95 \%$ Cl, 0.68-24.21) in the middle-income country subgroup.

Subgroup analysis by regions of the different risk level showed the pooled OR of MTCT was 9.48 ( $95 \% \mathrm{Cl}, 4.77-18.85)$ in the moderate risk region subgroup; in the high-risk region subgroup, the overall MTCT OR was 5.00 (95\% Cl: $0.17-146.64)$ and in the low-risk region subgroup, the overall MTCT OR was 6.01 (95\% Cl: 2.13-16.97).

Meta-regression analysis indicated that publication year $(\mathrm{Z}=0.13, P=0.897)$ and MTCT incidence $(\mathrm{Z}=0.92, P=0.359)$ were not correlate to HCV MTCT risk.

\section{Comparison of HCV MTCT incidence between different HCV-RNA titers}

Nine publications ${ }^{18,31,41-44,52-54}$ with 881 newborns of HCV infected mother met eligibility for comparing MTCT incidence between mothers with high ( $\geq 6$ log10 copies/ml) or low level of viremia (< 6 log copies $/ \mathrm{ml}$ ) (Table 2). The pooled MTCT incidence in high viremia group was 11.30\% (95\% Cl: $8.15 \%-15.47 \%$ ) and $2.38 \%$ (95\% Cl: $6.57 \%-27.95 \%)$ in low viremia group.

No significant heterogeneity observed across studies $\left(Q=9.28, P=0.320 ; I^{2}=0.00 \%\right)$. Pregnant women with high level of viremia experienced an elevated risk of HCV MTCT (OR=4.93, 95\% Cl: 2.38,-10.21; P<0.001) compared with those with low HCV viremia titer by random effects model analysis (Fig. 2B). We conducted further sensitivity analysis by limiting studies to those prospective studies with a sample size larger than 100, and the pooled OR of MTCT was 3.82 (95\% Cl: 1.35-10.79), indicating good robustness of the results (supplementary appendix, Fig. 1B).

The funnel plot showed symmetry, which indicated insufficient evidence of publication bias (supplementary appendix, Fig. 2B). Begg's test $(P=0.920)$ and Egger's test $(P=0.455)$ also suggested the absence of publication bias. 
Subgroup analysis by country income showed the pooled MTCT OR was 5.00 (95\% Cl: 2.54-11.24) in the high-income country subgroup, and 0.69 ( $95 \%$ Cl:0.02-26.90) in the middle-income country subgroup.

Subgroup analysis by region of the different risk level showed the pooled MTCT OR was $3.84(95 \% \mathrm{Cl}, 1.22-12.09)$ in the moderate risk region subgroup, and the overall MTCT OR was 7.21 (95\% Cl, 1.62-32.12) in the low-risk region subgroup.

Meta-regression analysis indicated that publication year $(Z=-0.27, P=0.784)$ and MTCT incidence $(Z=0.31, P=0.754)$ were not relevant to MTCT risk.

\section{Discussion}

The current meta-analysis, involving 4382 newborns with maternal HCV antibody positive, found a significantly increased risk of HCV MTCT in pregnant women with HCV viremia, and an even higher MTCT incidence in pregnant women with high level of viremia. In addition, we found that HIV coinfection further increase risk of MTCT in women with HCV viremia.

In spite of the clear association of HIV co-infection and HCV MTCV during pregnancy, HCV viremia was been confirmed as an independent risk factor HCV transmission according to the result of the present meta-analysis. Although HCV MTCT was found in 4 out of 1291 newborns with maternal HCV-RNA negative, this could be explained by variation of HCV RNA titers during pregnancy, or false negative results due to low sensitivity of testing kits ${ }^{33,42,49,51}$. In addition, mothers with HCV viremia titer greater than or equal to $6 \mathrm{log}$ copies/ml were with even higher incidence of MTCT than those with HCV viremia titer lower than 6 log copies/ml. It indicates pregnant women with HCV viremia should be considered for antiviral treatment and 6 log copies/ml coule be considered as the threshold for who is proposed to receive antiviral treatment. DAA treatment is an effective and safe strategy to reduce HCV RNA viral load in patient with chronic HCV infection ${ }^{55}$. Recently, DAA treatment with Ledipasvir and sofosbuvir regimens showed its effectiveness among pregnant women ${ }^{14}$. However, up to $79 \%$ women refused to receive DAA treatment during pregnancy, concerning the safety and cost of DAA ${ }^{56}$. The findings of this study suggested the emergency of DAA treatment for HCV infect pregnant women, and strengthening health promotion among pregnant women and reducing the cost of DAA are future measures to be taken.

Maternal HIV coinfection is considered as another risk factor of $\mathrm{MTCT}^{57}$. In this meta-analysis, pregnant women co-infected of HIV experience significantly highest prevalence of HCV-MTCT. This result is consistent with the report by Benova et al ${ }^{57}$. The role of HCV/HIV coinfection in HCV transmission seems to depend on HCV-RNA titer, since women taken HAART therapy, by reducing HIV-RNA level, also reduces the risk of HCV-MTCT ${ }^{18}$. Whereas, for the same level of HCV-RNA titer, the risk of HCV MTCT is probably higher in HCV/HIV co-infected women than HCV viremia mono-infected women according to the present meta-analysis. This suggests that other mechanisms could be involved to explain HCV-MTCT. Further studies are necessary to explore the interaction between HCV and HIV. Our findings suggested for HIV/HCV co-infected pregnant women antiviral treatments for both HIV and HCV virus could be considered; however, further clinical trials are needed to confirm the safety and effectiveness of the combined treatment.

Inevitably, the present meta-analysis has some limitations. First, because of data source, we were only able to include cohort study for analysis. Second, due to the heterogeneity of studies regarding to HCV viral load, the dose-response relationship between HCV RNA and MTCT could not be obtained. In addition, the difference of MTCT among diverse HCV gene type is not clear, because the gene type of HCV was not available in literature ${ }^{14}$. Although with these limitations, our study is the first meta-analysis to determine the association between HCV viral load and MTCT during pregnancy.

\section{Conclusion}

In conclusion, detectable HCV viremia is an independent factor of MTCT in pregnant women. HCV/HIV co-infection can further increase the risk of HCV-MTCT. The 6 log copies/ml of maternal HCV viral load may be considered as the threshold for MTCT. Further randomized controlled trails are needed to confirm the indication of DAA treatment in pregnant women.

\section{Abbreviations}

HCV: hepatitis C virus; HIV: human immunodeficiency virus; MTCT: mother-to-child transmission; OR : odds ratio; $\mathrm{Cl}=$ confidence interval; DAA: direct-acting antiviral; CENTRAL: the Cochrane Central Register of Controlled Trials; SCIE: Science Citation Index Expanded; CPCIS: Conference Proceedings Citation IndexScience ; LILACS: Literature Latino-Americana e do Caribe em Ciências da Saúde

\section{Declarations}

\section{Acknowledgements}

Not applicable.

\section{Authors' contributions}

Songqing Deng and Wenfang Zhong: project development, data management, data analysis, and manuscript writing. Chen Wen and Zilian Wang: manuscript revise and project development. All authors read and approved the final manuscript.

\section{Funding}

Not applicable. 
Availability of data and materials

All data can be obtained from the manuscript.

\section{Ethics approval and consent to participate}

Not applicable.

\section{Consent for publication}

Not applicable.

\section{Competing interests}

Not applicable.

\section{References}

1. Petruzziello A, Marigliano S, Loquercio G, Cozzolino A, Cacciapuoti C. Global epidemiology of hepatitis $C$ virus infection: An up-date of the distribution and circulation of hepatitis C virus genotypes. World J Gastroenterol. 2016;22(34):7824-40.

2. Meer AJvd, Veldt BJ, Feld JJ, Wedemeyer H, Dufour J-F, Lammert F et al. Association between sustained virological response and all-cause mortality among patients with chronic hepatitis $C$ and advanced hepatic fibrosis. JAMA. 2012;308(24):2584-93.

3. Society for Maternal-Fetal Medicine. Electronic address pso, Hughes BL, Page CM, Kuller JA. Hepatitis C in pregnancy: screening, treatment, and management. Am J Obstet Gynecol. 2017;217(5):B2-B12.

4. Ryerson AB, Schillie S, Barker LK, Kupronis BA, Wester C. Vital Signs: Newly Reported Acute and Chronic Hepatitis C Cases - United States, $2009-2018$. MMWR Morb Mortal Wkly Rep. 2020;69(14):399-404.

5. Koneru A, Nelson N, Hariri S, Canary L, Sanders KJ, Maxwell JF et al. Increased Hepatitis C Virus (HCV) Detection in Women of Childbearing Age and Potential Risk for Vertical Transmission - United States and Kentucky, 2011-2014. Mmwr-Morbid Mortal W. 2016;65(28):705-10.

6. Mok J, Pembrey L, Tovo P-A, Newell M-L, Network EPHCV. When does mother to child transmission of hepatitis C virus occur? Arch Dis Child Fetal Neonatal Ed. 2005;90(2):F156-60.

7. Tovo PA, Calitri C, Scolfaro C, Gabiano C, Garazzino S. Vertically acquired hepatitis C virus infection: Correlates of transmission and disease progression. World J Gastroenterol. 2016;22(4):1382-92.

8. Schillie S, Wester C, Osborne M, Wesolowski L, Ryerson AB. CDC Recommendations for Hepatitis C Screening Among Adults - United States, 2020. Mmwr Recomm Rep. 2020;69(2):1-17.

9. Force USPST, Owens DK, Davidson KW, Krist AH, Barry MJ, Cabana M et al. Screening for Hepatitis C Virus Infection in Adolescents and Adults: US Preventive Services Task Force Recommendation Statement. JAMA. 2020;323(10):970-5.

10. Society for Maternal-Fetal M, Dionne-Odom J, Tita AT, Silverman NS. \#38: Hepatitis B in pregnancy screening, treatment, and prevention of vertical transmission. Am J Obstet Gynecol. 2016;214(1):6-14.

11. Bailey H, Zash R, Rasi V, Thorne C. HIV treatment in pregnancy. Lancet HIV. 2018;5(8):e457-e67.

12. ACOG Practice Bulletin No.142: Cerclage for the management of cervical insufficiency. Obstet Gynecol 2014;123(2 Pt 1):372-9.

13. Lawitz E, Poordad FF, Pang PS. Sofosbuvir and ledipasvir fixed-dose combination with and without ribavirin in treatment-naive and previously treated patients with genotype 1 hepatitis C virus infection (LONESTAR): an open-label, randomised, phase 2 trial (vol 383, pg 515, 2014). Lancet. 2014;383(9920):870-

14. Chappell CA, Scarsi KK, Kirby BJ, Suri V, Gaggar A, Bogen DL et al. Ledipasvir plus sofosbuvir in pregnant women with hepatitis C virus infection: a phase 1 pharmacokinetic study. Lancet Microbe. 2020;1(5):e200-e8.

15. Tovo PA, Calitri C, Scolfaro C, Gabiano C, Garazzino S. Vertically acquired hepatitis C virus infection: Correlates of transmission and disease progression. World J Gastroentero. 2016;22(4):1382-92.

16. Delotte Jrm, Barjoan EnM, Berre'bi A, Laffont C, Benos P, Pradier C et al. Obstetric management does not influence vertical transmission of HCV infection: results of the ALHICE group study. J Matern Fetal Neonatal Med. 2014;27(7):664-70.

17. Ceci O, Margiotta M, Marello F, Francavilla R, Loizzi P, Francavilla A et al. Vertical transmission of hepatitis C virus in a cohort of 2,447 HIV-seronegative pregnant women: a 24-month prospective study. J Pediatr Gastroenterol Nutr. 2001;33(5):570-5.

18. Marine-Barjoan E, Berrebi A, Giordanengo V, Favre SF, Haas H, Moreigne M et al. HCV/HIV co-infection, HCV viral load and mode of delivery: risk factors for mother-to-child transmission of hepatitis C virus? AIDS. 2007;21(13):1811-5.

19. Vertical transmission of the hepatitis C virus: Current knowledge and issues. Paediatr Child Health. 2008;13(6):529-41.

20. Pott HJ, Theodoro M, de Almeida Vespoli J, Senise JF, Castelo A. Mother-to-child transmission of hepatitis C virus. Eur J Obstet Gynecol Reprod Biol. 2018;224:125-30.

21. Le Campion A, Larouche A, Fauteux-Daniel S, Soudeyns H. Pathogenesis of hepatitis C during pregnancy and childhood. Viruses. 2012;4(12):3531-50.

22. Jhaveri R, Hashem M, El-Kamary SS, Saleh DA, Sharaf SA, El-Mougy F et al. Hepatitis C Virus (HCV) Vertical Transmission in 12-Month-Old Infants Born to HCV-Infected Women and Assessment of Maternal Risk Factors. Open Forum Infect Dis. 2015;2(2):ofv089.

Page $10 / 13$ 
23. WHO (2017) Global Hepatitis Report. http://wwwwhoint/hepatitis/publications/global-hepatitis-report2017-executive-summary/en/

24. Morse A, 4th ASB, Jhaveri R. Individual State Hepatitis C Data Supports Expanding Screening Beyond Baby Boomers to All Adults. Gastroenterology. 2018;154(6):1850-1.e2.

25. Benhammou V, Tubiana R, Matheron S, Sellier P, Mandelbrot L, Chenadec JL et al. HBV or HCV Coinfection in HIV-1-Infected Pregnant Women in France: Prevalence and Pregnancy Outcomes. J Acquir Immune Defic Syndr. 2018;77(5):439-50.

26. Eyster ME, Fried MW, Di Bisceglie AM, Goedert JJ. Increasing hepatitis C virus RNA levels in hemophiliacs: relationship to human immunodeficiency virus infection and liver disease. Multicenter Hemophilia Cohort Study. Blood. 1994;84(4):1020-3.

27. Funk AL, Lu Y, Yoshida K, Zhao T, Boucheron P, van Holten J et al. Efficacy and safety of antiviral prophylaxis during pregnancy to prevent mother-to-child transmission of hepatitis B virus: a systematic review and meta-analysis. Lancet Infect Dis. 2021;21(1):70-84.

28. Bender R, Lange S. Adjusting for multiple testing-when and how? J Clin Epidemiol 2001;54(4):343-9.

29. S H. A simple sequentially rejective multiple test procedure. Scandinavian journal of statistics. 1979:65-70.

30. Bank. W. Country classification table.. 2020.

31. Saez A, Losa M, Lo lacono O, Lozano C, Alvarez E, Pita L et al. Diagnostic and prognostic value of virologic tests in vertical transmission of hepatitis C virus infection: results of a large prospective study in pregnant women. Hepatogastroenterology. 2004;51(58):1104-8.

32. Elmagd EkA, Abdel-wahab KS, Alrasheedy ZE, S.Khalifa A. An egyptian study of mother to child transmission of hepatitis c virus. international journal of virology. 2011;5(3):100-8.

33. Granovsky MO, Minkoff HL, Tess BH, Waters D, Hatzakis A, Devoid DE et al. Hepatitis C virus infection in the mothers and infants cohort study. Pediatrics. 1998;102(2 Pt 1):355-9.

34. Ngo-Giang-Huong N, Jourdain G, Sirirungsi W, Decker L, Khamduang W, Le Coeur S et al. Human immunodeficiency virus-hepatitis C virus co-infection in pregnant women and perinatal transmission to infants in Thailand. Int J Infect Dis. 2010;14(7):e602-7.

35. Steininger C, Kundi M, Jatzko G, Kiss H, Lischka A, Holzmann H. Increased risk of mother-to-infant transmission of hepatitis $\mathrm{C}$ virus by intrapartum infantile exposure to maternal blood. J Infect Dis. 2003;187(3):345-51.

36. Dal Molin G, D'Agaro P, Ansaldi F, Ciana G, Fertz C, Alberico S et al. Mother-to-infant transmission of hepatitis C virus: Rate of infection and assessment of viral load and IgM Anti-HCV as risk factors. J Med Virol. 2002;67(2):137-42.

37. Money D, Boucoiran I, Wagner E, Dobson S, Kennedy A, Lohn Z et al. Obstetrical and neonatal outcomes among women infected with hepatitis C and their infants. J Obstet Gynaecol Can 2014;36(9):785-94.

38. Mazza C, Ravaggi A, Rodella A, Padula D, Duse M, Lomini M et al. Prospective study of mother-to-infant transmission of hepatitis C virus (HCV) infection. Study Group for Vertical Transmission. J Med Virol. 1998;54(1):12-9.

39. Ferrero S, Lungaro P, Bruzzone BM, Gotta C, Bentivoglio G, Ragni N. Prospective study of mother-to-infant transmission of hepatitis C virus: a 10-year survey (1990-2000). Acta Obstet Gynecol Scand. 2003;82(3):229-34.

40. Murakami J, Nagata I, litsuka T, Okamoto M, Kaji S, Hoshika T et al. Risk factors for mother-to-child transmission of hepatitis $\mathrm{C}$ virus: Maternal high viral load and fetal exposure in the birth canal. Hepatol Res. 2012;42(7):648-57.

41. Mast EE, Hwang LY, Seto DS, Nolte FS, Nainan OV, Wurtzel H et al. Risk factors for perinatal transmission of hepatitis C virus (HCV) and the natural history of HCV infection acquired in infancy. J Infect Dis. 2005;192(11):1880-9.

42. Garcia-Tejedor A, Maiques-Montesinos V, Diago-Almela VJ, Pereda-Perez A, Alberola-Cunat V, Lopez-Hontangas JL et al. Risk factors for vertical transmission of hepatitis C virus: a single center experience with 710 HCV-infected mothers. Eur J Obstet Gynecol Reprod Biol. 2015;194:173-7.

43. Ohto H, Terazawa S, Sasaki N, Sasaki N, Hino K, Ishiwata C et al. Transmission of hepatitis C virus from mothers to infants. The Vertical Transmission of Hepatitis C Virus Collaborative Study Group. N Engl J Med. 1994;330(11):744-50.

44. Spencer JD, Latt N, Beeby PJ, Collins E, Saunders JB, McCaughan GW et al. Transmission of hepatitis C virus to infants of human immunodeficiency virus-negative intravenous drug-using mothers: rate of infection and assessment of risk factors for transmission. J Viral Hepat 1997;4(6):395-409.

45. Ruiz-Extremera A, Munoz-Gamez JA, Abril-Molina A, Salmeron-Ruiz MA, Munoz-de-Rueda P, Pavon-Castillero EJ et al. Variation of transaminases, HCVRNA levels and Th1/Th2 cytokine production during the post-partum period in pregnant women with chronic hepatitis C. PLoS One. 2013;8(10):e75613.

46. Resti M, Azzari C, Mannelli F, Moriondo M, Novembre E, de Martino M et al. Mother to child transmission of hepatitis $C$ virus: prospective study of risk factors and timing of infection in children born to women seronegative for HIV-1. Tuscany Study Group on Hepatitis C Virus Infection. BMJ. 1998;317(7156):437-41.

47. Zuccotti GV, Ribero ML, Giovannini M, Fasola M, Riva E, Portera G et al. Effect of hepatitis C genotype on mother-to-infant transmission of virus. J Pediatr. 1995;127(2):278-80.

48. Zanetti AR, Tanzi E, Romano L, Zuin G, Minola E, Vecchi L et al. A prospective study on mother-to-infant transmission of hepatitis C virus. Intervirology. 1998;41(4-5):208-12.

49. Resti M, Azzari C, Galli L, Zuin G, Giacchino R, Bortolotti F et al. Maternal drug use is a preeminent risk factor for mother-to-child hepatitis $C$ virus transmission: results from a multicenter study of 1372 mother-infant pairs. J Infect Dis 2002;185(5):567-72.

50. Tajiri H, Miyoshi Y, Funada S, Etani Y, Abe J, Onodera T et al. Prospective study of mother-to-infant transmission of hepatitis $\mathrm{C}$ virus. Pediatr Infect Dis J. 2001;20(1):10-4.

51. Paccagnini S, Principi N, Massironi E, Tanzi E, Romano L, Muggiasca ML et al. Perinatal transmission and manifestation of hepatitis C virus infection in a high risk population. Pediatr Infect Dis J. 1995;14(3):195-9.

Page 11/13 
52. Okamoto M, Nagata I, Murakami J, Kaji S, litsuka T, Hoshika T et al. Prospective reevaluation of risk factors in mother-to-child transmission of hepatitis C virus: high virus load, vaginal delivery, and negative anti-NS4 antibody. J Infect Dis. 2000;182(5):1511-4.

53. Ketzinel-Gilad M, Colodner SL, Hadary R, Granot E, Shouval D, Galun E. Transient transmission of hepatitis C virus from mothers to newborns. Eur J Clin Microbiol Infect Dis. 2000;19(4):267-74.

54. Lin HH, Kao JH, Hsu HY, Ni YH, Yeh SH, Hwang LH et al. Possible role of high-titer maternal viremia in perinatal transmission of hepatitis $\mathrm{C}$ virus. $\mathrm{J}$ Infect Dis. 1994;169(3):638-41.

55. Falade-Nwulia O, Suarez-Cuervo C, Nelson DR, Fried MW, Segal JB, Sulkowski MS. Oral Direct-Acting Agent Therapy for Hepatitis C Virus Infection: A Systematic Review. Ann Intern Med. 2017;166(9):637-48.

56. Kushner T, Cohen J, Tien PC, Terrault NA. Evaluating Women's Preferences for Hepatitis C Treatment During Pregnancy. Hepatol Commun. 2018;2(11):1306-10.

57. Benova L, Mohamoud YA, Calvert C, Abu-Raddad LJ. Vertical Transmission of Hepatitis C Virus: Systematic Review and Meta-analysis. Clin Infect Dis. 2014;59(6):765-73.

\section{Figures}

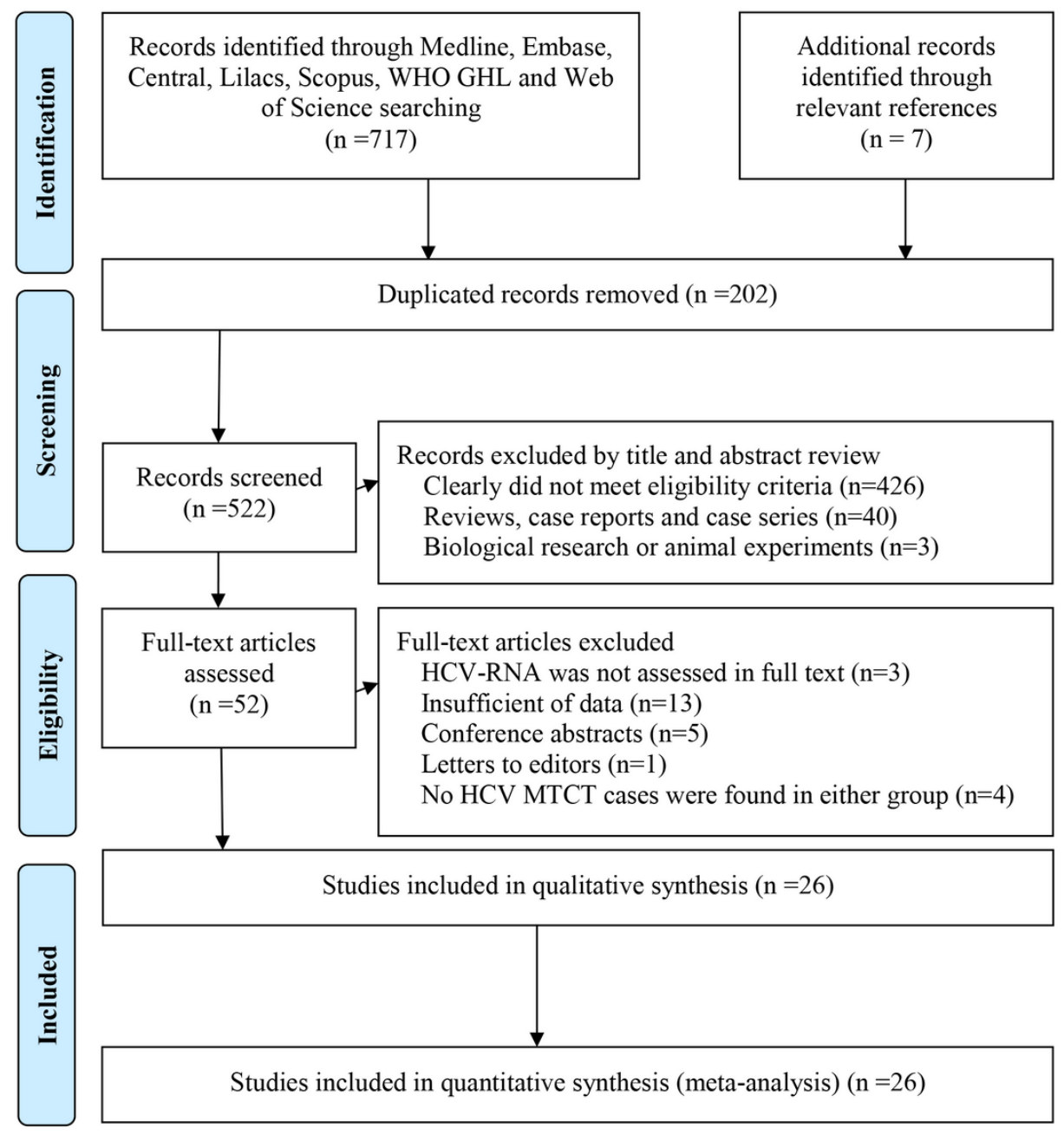

Figure 1

Flowchart of selection of studies included in meta-analysis 

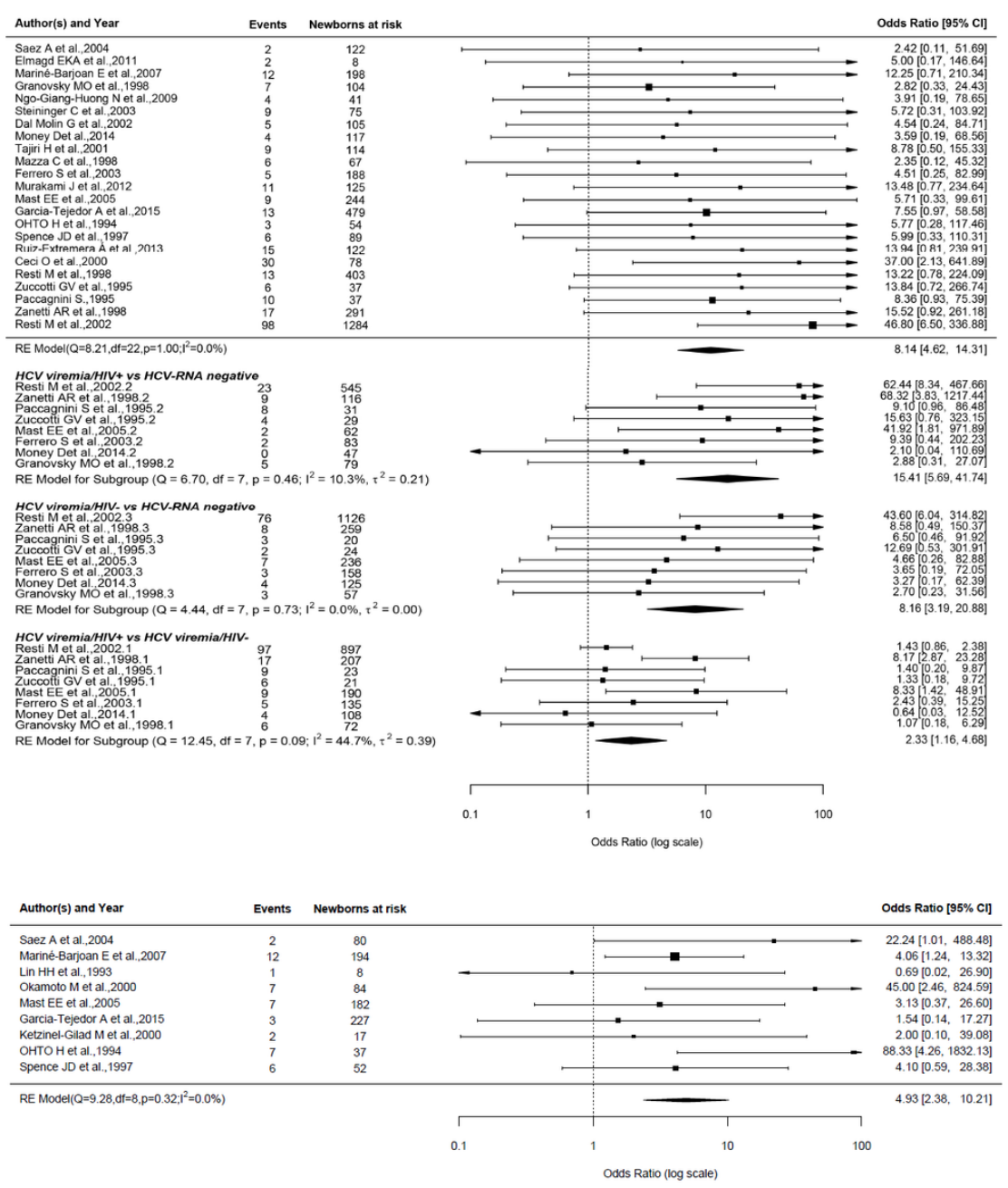

\section{Figure 2}

A Forest plot of MTCT risk of maternal HCV viremia group compared with HCV-RNA-negative group. MTCT= mother-to-child transmission, HCV=Hepatitis C virus B Forest plot of MTCT incidence for maternal HCV-RNA titer $\geq 6$ log copies $/ \mathrm{mL}$ Compared with $<6$ log copies $/ \mathrm{ml}$. MTCT= mother-to-child transmission, $\mathrm{HCV}=$ Hepatitis $\mathrm{C}$ virus

\section{Supplementary Files}

This is a list of supplementary files associated with this preprint. Click to download.
- supplementaryappendixFig1A.pdf
- supplementaryappendixFig1B.pdf
- supplementaryappendixFig2A.pdf
- supplementaryappendixFig2B.pdf
- SupplementaryFigureLegends.docx
- supplementaryTable1.docx
- supplementaryTable2.docx
- supplementarytable3.docx
- supplementaryappendistable4.docx
- supplementaryappendixFig3A.pdf
- supplementaryappendixFig3B.pdf
- supplementaryappendixFig4A.pdf
- supplementaryappendixFig4B.pdf 